J. Nonlinear Var. Anal. 4 (2020), No. 2, pp. 241-252

Available online at http://jnva.biemdas.com

https://doi.org/10.23952/jnva.4.2020.2.06

\title{
AN EXTREMAL VECTOR PROBLEM UNDER INCLUSION CONSTRAINTS VIA RADIAL EPI-DERIVATIVES
}

\author{
MOHAMED AIT MANSOUR ${ }^{1, *}$, ENNOURI TAZI $^{2}$ \\ ${ }^{1}$ Département de Physiques, LPFAS, Faculté Poly-disciplinaire, Safi, Université Cadi Ayyad, Morocco \\ ${ }^{2}$ Département de Mathématiques, Faculté des Sciences, Université Chouaib Doukkali, El Jadida, Morocco
}

\begin{abstract}
In this paper, we consider an extremal problem under inclusions constraints. Based on recent semicontinuous epi-derivatives results, we establish necessary and sufficient optimality conditions of Fermat type for this problem. We do not require any differentiability or regularity condition on the objective function. We finally give some examples of functions to which our results may be applied.
\end{abstract}

Keywords. Extremal vector problem; Inclusion constraint; Radial epi-derivatives; Optimality conditions; Starshaped functions.

\section{INTRODUCTION}

In this paper, we are concerned with optimality conditions of Fermat type for the following vector optimization problems under an inclusion constraint:

$$
\text { Maximize } f(x) \text { subject to } 0 \in F(x) \text {, }
$$

where $f: X \longrightarrow Y^{\bullet}$ is an extended vector-valued mapping with $X$ being a reflexive Banach space, $Y$ being a reflexive Banach space ordered by a closed, convex and proper cone $P$, and $F: X \longrightarrow 2^{Z}$ is a set-valued map from $X$ to another reflexive Banach space $Z$.

A point $\bar{x} \in \operatorname{Dom}(f)$ is a solution to (P) if and only if $0 \in F(\bar{x})$ and

$$
f(x) \leq_{P} f(\bar{x}) \text { for all } x \in \operatorname{dom}(f) \text { s.t } 0 \in F(x) .
$$

In [1], Amahroq, Gadhi and Riahi provided first order optimality conditions in the case where $f$ is an extended real function. The basic tool used there is the classical epi-differentiability of $f$ based on the concept of Mosco-epi-convergence Attouch [6] that allows to define the epiderivative of extended real functions, which was introduced in Rockafellar [20]. In the vector case, i.e., the target space of $f$ is a partially ordered vector space, it is not easy to define an adequate epi-convergence having the same properties of the scalar case. It seems that this reason motivated Bazán [7] to propose, as an efficient alternative, the radial epi-derivatives of vector-valued maps ranging in a order-complete space. This approach does not necessities any convergence notion for sequences of extended vector-valued maps and allows to obtain optimality conditions for the large class of nonconvex functions.

${ }^{*}$ Corresponding author.

E-mail addresses: ait.mansour.mohamed@gmail.com (M. Ait Mansour), taznouri@gmail.com (E. Tazi).

Received March 11, 2020; Accepted May 23, 2020.

(C)2020 Journal of Nonlinear and Variational Analysis 
In this paper, to establish the optimality conditions for problem $(\mathrm{P})$, we rather make appeal to the recent semicontinuous extended radial epi-derivatives introduced and studied in [2, 3]. The semicontinuity of these derivatives allows us, on the one hand, to improve and extend to the vector case the results presented in [1], and on the other hand, enables us to provide the link between the radial epi-derivatives and Mosco epi-derivatives of star-shaped epi-differentiable extended real-valued functions. An entirely related reference to the topic of this paper is the recent book by Khan, Tammer and Zălinescu [15], which contains the mostly important background material and provides a detailed discussion of optimality conditions. For more information on the use of epi-derivaties and related analysis, we refer to $[5,10,11,12,13,14,16,17,21]$ and the references therein.

The paper is organized as follows. In Section 2, we fix the notation and collect the necessary background materials to establish our main results. In Section 3, we present a general class of functions for which radial epi-derivatives coincide with Mosco epi-derivatives. In Section 4, we consider an extremal vector problem for which we obtain both necessary and sufficient optimality conditions. The paper is ended by a concluding section, Section 5 .

\section{BACKGROUND MATERIALS}

Throughout this paper, $Y$ denotes a reflexive Banach space. For any subset $A$ of $Y, \operatorname{cl}(A)$ will stand for its closure, $\operatorname{Int}(A)$ its interior and $A^{c}$ its complement.

We require that $Y$ is ordered by a closed, convex and proper cone $P$ (i.e., $\{0\} \neq P \neq Y$ ). The cone $P$ defines a partial ordering on $Y$ denoted by $\leq_{P}$ and given by

$$
\left.y_{1} \leq_{P} y_{2} \text { (or equivalently } y_{2} \geq_{P} y_{1}\right) \Longleftrightarrow y_{2} \in y_{1}+P \text {. }
$$

If needed, the cone $P$ will be supposed, in addition, to be pointed (i.e., $l(P):=P \cap(-P)=\{0\}$ ). We adjoin to $Y$ two artificial elements $-\infty$ and $+\infty$ such that

$$
-\infty \leq_{P} y \leq_{P}+\infty
$$

for all $y \in Y$. We denote by $\bar{Y}$ the extended space $Y \cup\{ \pm \infty\}$ and assume the following conventions:

- $( \pm \infty)+y=y+( \pm \infty)= \pm \infty$ for all $y \in Y$;

- $( \pm \infty)+( \pm \infty)=( \pm \infty)$;

- $\lambda( \pm \infty)=( \pm \infty)$ for all $\lambda>0$, and $\lambda( \pm \infty)=\mp$ for all $\lambda<0$.

$Y^{\bullet}$ stands for $Y \cup\{+\infty\}$, that is, $Y^{\bullet}=Y \cup\{+\infty\}$. $P^{\bullet}$ stands for $P \cup\{+\infty\}$, that is, $P^{\bullet}=$ $P \cup\{+\infty\}$. Int $P^{\bullet}$ stands for $\operatorname{Int}(P) \cup\{+\infty\}$, that is, $\operatorname{Int} P^{\bullet}=\operatorname{Int}(P) \cup\{+\infty\}$.

A new ordering is then defined on $\bar{Y}$ by $x \leq P_{\bullet} y \Leftrightarrow y-x \in P^{\bullet}$, for $x, y \in \bar{Y}$. We will write $x<_{P \bullet} y$ if $y-x \in \operatorname{Int} P^{\bullet}$. If the set of $P$-upper bounds (resp. $P$-lower bounds) of a $P$-upper bounded (resp. $P$-lower bounded) subset $A \subset Y$ admits a least (resp. greatest) element, it is called a $P$-supremum (resp. $P$-infimum) of $A$. The set of $P$-supremums (resp. $P$-infimums) of $A$ is denoted by $\sup _{P}(A)\left(\operatorname{resp} \inf _{P}(A)\right)$.

If a subset $A \subset Y$ admits a $P$-upper bound (resp. $P$-lower bound) $a$ in $A(a \in A)$, then, $a$ is called a $P$-greatest element or $P$-maximum (resp. least element or minimum) of $A$. The set of $P$-maximums (resp. $P$-minimums) of $A$ is denoted by $\max _{P}(A)\left(\right.$ resp. $\min _{P}(A)$.) 
The cone $P$ will supposed to be normal, i.e., the origin 0 admits a base $\mathscr{B}$ of neighborhoods such that

$$
(V-P) \cap(V+P)=V, \forall V \in \mathscr{B} .
$$

2.1. Semicontinuity. Consider another reflexive Banach $X$. For any point $u$ in $X$ or $Y$, we denote by $\vartheta(u)$ the family of neighborhoods of $u$. For an extended-vector-valued mapping $f$ : $X \rightarrow Y^{\bullet}$, the following notations and definitions will also be needed:

- The domain :

$$
\operatorname{Dom}(f)=\{x \in X \mid f(x) \in Y\}
$$

- The epigraph:

epi $(f)=\left\{(x, y) \in X \times Y \mid x \in \operatorname{Dom}(f), f(x) \leq_{P} y\right\} \cup\{(x, y) \in X \times Y \mid, f(x)=-\infty\}$;

- The hypograph:

$\operatorname{hyp}(f)=\left\{(x, y) \in X \times Y \mid x \in \operatorname{Dom}(f), y \leq_{P} f(x)\right\} \cup\{(x, y) \in X \times Y \mid, f(x)=+\infty\}$;

- The graph:

$$
\operatorname{gph}(f)=\{(x, y) \in X \times Y \mid x \in \operatorname{Dom}(f), y=f(x)\} .
$$

For a set-valued map $S: X \longrightarrow 2^{Y}$, i.e., a mapping which assigns to every $x \in Y$ a (possibly empty) subset of $Y$, we recall:

- The domain:

$$
\operatorname{Dom}(S)=\{x \in X \mid S(x) \neq \emptyset\}
$$

- The graph:

$$
\operatorname{gph}(S)=\{(x, y) \in X \times Y \mid x \in \operatorname{Dom} S, y \in S(x)\} ;
$$

We now recall the notion of the semicontinuity of vector-valued maps which can be found, among others, in $[8,9,19]$.

- A mapping $f: X \rightarrow Y^{\bullet}$ is said to be lower semicontinuous ( $P-1$.s.c) at $\bar{x} \in X$ if, for any neighborhood $V$ of zero and for any $y \in Y$ satisfying $y \leq_{P} f(\bar{x})$, there exists a neighborhood $U$ of $\bar{x}$ in $X$ such that

$$
f(U) \subset y+V+P^{\bullet} .
$$

- The mapping $f: X \rightarrow Y^{\bullet}$ is said to be sequentially lower semicontinuous (s-l.s.c) at $\bar{x} \in X$ if, for any $y \in Y$ satisfying $y \leq_{P} f(\bar{x})$ and for any sequence $\left(x_{n}\right)$ converging to $\bar{x}$, there exists a sequence $\left(y_{n}\right)$ converging to $y$ such that $y_{n} \leq_{P} f\left(x_{n}\right)$, for every $n$.

- If $\bar{x} \in \operatorname{Dom}(f)$, then $f$ is s-l.s.c at $\bar{x}$ if and only if, for each sequence $\left(x_{n}\right)$ converging to $\bar{x}$, there exists a sequence $\left(y_{n}\right)$ converging to $f(\bar{x})$ such that $y_{n} \leq_{P} f\left(x_{n}\right)$ for all $n$.

- Since $X$ and $Y$ are normed spaces, then $f$ is $P$-l.s.c at $\bar{x}$ if, and only if $f$ is s-l.s.c at $\bar{x}$.

- $f$ is said to be sequentially upper semicontinuous ( s-u.s.c for short) if and only if $-f$ is s-l.s.c.

Now for a subset $C$ in a normed space $E$, at a point $\bar{x} \in \operatorname{cl}(C)$, the following concepts will be considered:

- The contingent (or tangent cone of Bouligand) cone:

$$
T(C, \bar{x})=\left\{v \in E \mid \exists t_{n} \searrow 0, \exists v_{n} \rightarrow v: \bar{x}+t_{n} v_{n} \in C, \forall n\right\} ;
$$


- The closed radial cone :

$$
R(C, \bar{x})=\left\{v \in E \mid \exists t_{n}>0, \exists v_{n} \rightarrow v: \bar{x}+t_{n} v_{n} \in C, \forall n\right\}
$$

2.2. The hypographical level set and regularization. Let us now collect some fundamental tools on semicontinuous regularizations and their related level sets from [4] (see also the recent work [3]).

For a given extended vector-valued map $g: X \rightarrow \bar{Y}=Y \cup\{-\infty,+\infty\}$ and a point $\bar{x} \in \operatorname{Dom}(g)$, we consider the following lower (resp. upper) semicontinuous regularization (introduced in [4] and refined in [3]) of $g$ at $\bar{x}$ :

$$
\begin{gathered}
v-\liminf _{x \rightarrow \bar{x}} g(x):=\max _{P}\left(A_{\bar{x}}^{g}\right):=\underline{g}(\bar{x}) \\
\left(\text { resp.v }-\limsup _{x \rightarrow \bar{x}} g(x):=\min _{P}\left(B_{\bar{x}}^{g}\right):=\bar{g}(\bar{x})\right),
\end{gathered}
$$

where $A_{\bar{x}}^{g}$ (resp. $B_{\bar{x}}^{g}$ ) is the lower (resp. upper) hypo (resp. epi)-graphical level set defined by

$$
\begin{gathered}
A_{\bar{x}}^{g}=\left\{y \in Y \mid \forall V \in \vartheta(y), \exists U \in \vartheta(\bar{x}), g(U) \subset V+P^{\bullet}\right\} \\
\text { (resp. } \left.B_{\bar{x}}^{g}=\left\{y \in Y \mid \forall V \in \vartheta(y), \exists U \in \vartheta(\bar{x}), g(U) \subset V-P^{\bullet}\right\}\right) .
\end{gathered}
$$

Theorem 2.1. [3, Theorem 2]

Let $f: X \longrightarrow Y^{\bullet}$ and $\bar{x} \in \operatorname{Dom}(f)$. Assume that the cone $P$ is normal and $Y$ is a lattice. Then,

i) If $f$ is $P$-lower bounded around $\bar{x}$, then $A_{\bar{x}}^{f}$ admits a unique $P$-maximum element $a_{\bar{x}} \in A_{\bar{x}}^{f}$ such that $\max _{P}\left(A_{\bar{x}}^{f}\right)=\left\{a_{\bar{x}}\right\}$.

ii) $f$ is $P$-upper bounded around $\bar{x}$, then $B_{\bar{x}}^{f}$ admits a unique $P$-minimum element $b_{\bar{x}} \in B_{\bar{x}}^{f}$ such that $\min _{P}\left(B_{\bar{x}}^{f}\right)=\left\{b_{\bar{x}}\right\}$.

Thanks to Theorem 2.1, for a given $f: X \longrightarrow Y^{\bullet}$, one finds the definition of [3] where the definitions of the lower and upper regularizations $\underline{f}$ and $\bar{f}$ are given by:

$$
\underline{f}(\bar{x}):=v-\liminf _{x \rightarrow \bar{x}} f(x)= \begin{cases}a_{\bar{x}}=\max _{P}\left(A_{\bar{x}}^{f}\right), & \text { if } \bar{x} \in \underline{D}(f), \\ -\infty, & \text { if } \bar{x} \in \operatorname{Dom}(f) \cap(\underline{D}(f))^{c}, \\ +\infty, & \text { if } \bar{x} \notin \operatorname{Dom}(f),\end{cases}
$$

where $\underline{D}(f)=\{x \in \operatorname{Dom}(f)$ and $f$ is $P$ - lower bounded around $x\}$, and

$$
\bar{f}(\bar{x}):=v-\limsup _{x \rightarrow \bar{x}} f(x)= \begin{cases}b_{\bar{x}}=\min _{P}\left(B_{\bar{x}}^{f}\right), & \text { if } \bar{x} \in \bar{D}(f), \\ +\infty, & \text { if } \bar{x} \in \operatorname{Dom}(f) \cap(\bar{D}(f))^{c}, \\ +\infty, & \text { if } \bar{x} \notin \operatorname{Dom}(f),\end{cases}
$$

where $\bar{D}(f)=\{x \in \operatorname{Dom}(f)$ and $f$ is $P$ - upper bounded around $x\}$. 
2.3. Radial epi-derivatives. To recall the radial epiderivatives introduced in [3], for a given extended vector-valued map $f: X \rightarrow \bar{Y}$, we first fix the following notations:

- $\left(\Delta_{t} f\right)_{\bar{x}}():.=t^{-1}(f(\bar{x}+t)-.f(\bar{x})) ; \quad t>0$;

- $f_{-}^{\prime}(\bar{x} ; u)=\inf _{t>0}\left(\Delta_{t} f\right)_{\bar{x}}(u)$;

- $f_{+}^{\prime}(\bar{x} ; u)=\sup _{t>0}\left(\Delta_{t} f\right)_{\bar{x}}(u)$.

Then, we define at $\bar{x} \in \operatorname{Dom}(f)$ the

- lower radial epiderivative of $f$ by

$$
\begin{aligned}
\underline{D}_{e}^{R} f(\bar{x} ; .): X & \longrightarrow \bar{Y} \\
u & \longmapsto v-\liminf _{u^{\prime} \rightarrow u} f_{-}^{\prime}\left(\bar{x} ; u^{\prime}\right) ;
\end{aligned}
$$

- upper radial epiderivative of $f$ by

$$
\begin{aligned}
\bar{D}_{e}^{R} f(\bar{x} ; .): X & \longrightarrow Y^{\bullet} \\
u & \longmapsto v-\limsup _{u^{\prime} \rightarrow u} f_{+}^{\prime}\left(\bar{x} ; u^{\prime}\right) .
\end{aligned}
$$

Indeed, with the following notations, we are able to give a complete definition:

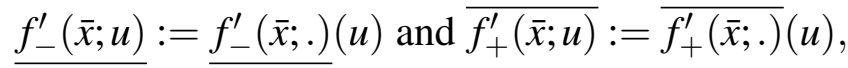

$$
\begin{aligned}
& \underline{D}_{e}^{R} f(\bar{x} ; u)= \begin{cases}f_{-}^{\prime}(\bar{x} ; u), & \text { if } u \in \underline{D}\left(f_{-}^{\prime}(\bar{x} ; .)\right), \\
\hline-\infty, & \text { if } u \in \operatorname{Dom}\left(f_{-}^{\prime}(\bar{x} ; .)\right) \cap\left(\bar{D}\left(f_{-}^{\prime}(\bar{x} ; .)\right)\right)^{c}, \\
+\infty, & \text { if } u \notin \operatorname{Dom}\left(f_{-}^{\prime}(\bar{x} ; .)\right),\end{cases}
\end{aligned}
$$

and

$$
\bar{D}_{e}^{R} f(\bar{x} ; u)= \begin{cases}\overline{f_{+}^{\prime}(\bar{x} ; u),} & \text { if } u \in \bar{D}\left(f_{+}^{\prime}(\bar{x} ; .)\right) \\ +\infty, & \text { if } u \in \operatorname{Dom}\left(f_{+}^{\prime}(\bar{x} ; .)\right) \cap\left(\bar{D}\left(f_{-}^{\prime}(\bar{x} ; .)\right)\right)^{c} \\ +\infty, & \text { if } u \notin \operatorname{Dom}\left(f_{+}^{\prime}(\bar{x} ; .)\right)\end{cases}
$$

where

$$
\underline{D}\left(f_{-}^{\prime}(\bar{x} ; .)\right)=\left\{u \in \operatorname{Dom}\left(f_{-}^{\prime}(\bar{x} ; .)\right) \text { and } f_{-}^{\prime}(\bar{x} ; .) \text { is } P-\text { lower bounded around } u\right\}
$$

and

$$
\bar{D}\left(f_{+}^{\prime}(\bar{x} ; .)\right)=\left\{u \in \operatorname{Dom}\left(f_{+}^{\prime}(\bar{x} ; .)\right) \text { and } f_{+}^{\prime}(\bar{x} ; .) \text { is } P \text { - upper bounded around } u\right\} .
$$

In other words, for any $\bar{x} \in \operatorname{Dom}(f)$, we have

$$
\underline{D}_{e}^{R} f(\bar{x} ; u)=\max _{P}\left(A_{u}^{f_{-}^{\prime}(\bar{x} ; .)}\right),
$$

and

$$
\bar{D}_{e}^{R} f(\bar{x} ; u)=\min _{P}\left(B_{u}^{f_{+}^{\prime}(\bar{x} ; \cdot)}\right) .
$$

Lemma 2.1. For all $\bar{x} \in \operatorname{Dom}(f), u \in X$, every time we have

$$
\underline{D}_{e}^{R} f(\bar{x} ; u) \leq_{P} f_{-}^{\prime}(\bar{x} ; u) \leq_{P} f_{+}^{\prime}(\bar{x} ; u) \leq_{P} \bar{D}_{e}^{R} f(\bar{x} ; u) .
$$

Proof. It is straightforward from [4, Proposition 5.1] and the definition of the radial epi-derivatives. 
Theorem 2.2. [3, Theorem 7] Assume that $P$ is normal such that $(Y, P)$ is a lattice space and $\operatorname{Int}(P) \neq \emptyset$. Let $f: X \longrightarrow Y^{\bullet}$. Then, for any $\bar{x} \in \operatorname{Dom}(f)$, we have

(a) the lower epiderivative $\underline{D}_{e}^{R} f(\bar{x} ;$.$) is positively homogenous i.e., for all u \in X$, for all $\lambda>0, \underline{D}_{e}^{R} f(\bar{x} ; \lambda u)=\lambda \underline{D}_{e}^{R} f(\bar{x} ; u)$

(b) $\underline{D}_{e}^{R} f(\bar{x} ; 0)=0 \Longleftrightarrow \underline{D}_{e}^{R} f(\bar{x} ; u) \neq-\infty, \forall u \in X$;

(c) the upper epiderivative $\bar{D}_{e}^{R} f(\bar{x} ;$.$) is positively homogenous i.e., for all u \in X$, for all $\lambda>0, \bar{D}_{e}^{R} f(\bar{x}, \lambda u)=\lambda \bar{D}_{e}^{R} f(\bar{x} ; u) ;$

(d) $\bar{D}_{e}^{R} f(\bar{x} ; 0)=0 \Longleftrightarrow \bar{D}_{e}^{R} f(\bar{x} ; u) \in Y, \forall u \in X$.

Theorem 2.3. Let $f: X \longrightarrow Y^{\bullet}, \bar{x} \in \operatorname{Dom}(f) ; \bar{y}=f(\bar{x})$ and $u \in X$. Assume that $P$ is normal such that $(Y, P)$ is a lattice space and $\operatorname{Int}(P) \neq \emptyset$. Then,

i) $\underline{D}_{e}^{R} f(\bar{x} ; u) \leq_{P} \inf _{P}\{v \in Y:(u, v) \in R(\operatorname{epi}(f),(\bar{x}, \bar{y}))\}$;

ii) $\left.\sup _{P}\{v \in Y:(u, v) \in R(\operatorname{hyp}(f),(\bar{x}, \bar{y}))\} \leq_{P} \bar{D}_{e}^{R} f(\bar{x} ; u)\right\}$.

If $Y=\mathbb{R}$ and $P=[0,+\infty[$, then equalities hold in $i)$ and $i i)$.

Proof. It is direct from [3, Theorem 10 and Remark 13].

\section{RADIAL EPIDERIVATIVES AND MOSCO-EPIDERIVATIVES}

The radial epiderivatives are well determined in the classic convex case (see, for instance, [3]), we reefer also to [7] wherein further examples are provided. Here we present another (general) example in which we link the radial epi-derivative to the Mosco-epiderivative of starshaped functions in the case of $Y=\mathbb{R}$. Assume, as before, that $X$ is a reflexive Banach space. We will write $\rightarrow$ for strong convergence and $\rightarrow$ for the weak convergence in $X$. Recall that a sequence $\left(\varphi_{n}\right)$ of extended real-valued functions from $X$ to $\mathbb{R} \cup\{+\infty\}$ is said to be Mosco-epiconvergent to $\varphi: X \rightarrow \mathbb{R} \cup\{+\infty\}$ if and only if, for all $x \in X$,

$$
\begin{cases}\text { i) } \forall x_{n} \rightarrow x & \varphi(x) \leq \liminf _{n \rightarrow+\infty} \varphi_{n}\left(x_{n}\right), \\ \text { ii) } \exists x_{n} \rightarrow x & \varphi(x) \geq \limsup _{n \rightarrow+\infty} \varphi_{n}\left(x_{n}\right) .\end{cases}
$$

If only the lower (resp. upper) part of this convergence (condition i)) (resp. ii)) is satisfied, we will say that $\varphi_{n}$ is lower (resp. upper) epi-convergent to $\varphi$. A family $\left(\varphi_{t}\right)_{t>0}$ of extendedreal functions from $X$ into $\mathbb{R} \cup\{+\infty\}$ is said to be Mosco-epi-convergent to $\varphi: X \rightarrow \mathbb{R} \cup\{+\infty\}$ if and only if, for every sequence $t_{n} \searrow 0$, the sequence $\left(\varphi_{t_{n}}\right)_{n}$ is Mosco-epi-convergent to $\varphi$. The function $\varphi$ is called in this case the Mosco-epi-limit of $\varphi$. If only $\left(\Delta_{t} f\right)_{\bar{x}}(.)_{t>0}$ lower (resp. upper) Mosco-epi-converges, we speak about lower (resp. upper) Mosco-derivative. A function $f: X \rightarrow \mathbb{R} \cup\{+\infty\}$ is said to be (Mosco-) epi-differentiable at a point $\bar{x} \in \operatorname{Dom}(f)$ if and only if the difference quotient functions $\left(\Delta_{t} f\right)_{\bar{x}}(.)_{t>0}$ Mosco-epi-converges and the Mosco-epi-limit (Mosco-epi-derivative) denoted by $f_{\bar{x}}^{\prime}$ satisfies $f_{\bar{x}}^{\prime}(0)>-\infty$. In this case, we denote by $\partial_{e} f(\bar{x})$ the epi-gradient of $f$ at $\bar{x}$, that is the set of all vectors $x^{\star} \in X^{\star}$ such that $\left\langle x^{\star}, v\right\rangle \leq f_{\bar{x}}^{\prime}(v), \forall v \in X$.

A function $f: X \rightarrow \mathbb{R} \cup\{+\infty\}$ is said to be lower (resp. upper) Mosco-epi-differentiable at a point $\bar{x} \in \operatorname{Dom}(f)$ if and only if the difference quotient functions $\left(\Delta_{t} f\right)_{\bar{x}}(.)_{t>0}$ lower (resp. upper) Mosco-epi-converges, we speak in this case about lower (resp. upper) Mosco-epi derivative. 
Definition 3.1. A subset $K$ in any normed space is called star-shaped w.r.t some point $\bar{x} \in K$ if $\bar{x}+t(x-\bar{x}) \in K$ for all $t \in[0,1]$ and all $x \in K$. A function $f: X \rightarrow \mathbb{R} \cup\{+\infty\}$ is said to be star-shaped w.r.t some point $\bar{x} \in \operatorname{Dom}(f)$ if and only if its epigraph is star-shaped at $(\bar{x}, f(\bar{x}))$.

Lemma 3.1. A function $f: X \rightarrow \mathbb{R} \cup\{+\infty\}$ is star-shaped at $x \in \operatorname{Dom}(f)$ if and only if there exists a number $\gamma>f(x)$ such that

$$
\lambda f(y)+(1-\lambda) \gamma \geq f(\lambda y+(1-\lambda) x), y \in \operatorname{Dom}(f), \lambda \in[0,1] .
$$

From this definition, we have the following characterization.

Lemma 3.2. Let $f: X \rightarrow \mathbb{R} \cup\{+\infty\}$ be star-shaped function at $\bar{x} \in \operatorname{Dom}(f)$. Then the closed radial and the contingent cones of epi $(f)$ at $(\bar{x}, f(\bar{x}))$ coincide, i.e.,

$$
R(\operatorname{epi}(f) ;(\bar{x}, f(\bar{x})))=T(\operatorname{epi}(f) ;(\bar{x}, f(\bar{x}))) .
$$

Proof. It is the consequence of $(b)$ of [7, Proposition 2.4].

Remark 3.1. It is known that, in particular, if $f$ is convex and the subdifferential $\partial f(\bar{x})$ (in the sense of convex analysis) of $f$ at $\bar{x}$ is nonempty, then $\underline{D}_{e}^{R} f(\bar{x} ; u)=f_{\bar{x}}^{\prime}(u)$.

The following Proposition extends this result to star-shaped functions.

Proposition 3.1. Let $f: X \rightarrow \mathbb{R} \cup\{+\infty\}$. The following assertions hold.

(i) If $f$ is upper epi-differentiable at a point $\bar{x} \in \operatorname{Dom}(f)$, then $\underline{D}_{e}^{R} f(\bar{x} ; u) \leq f_{\bar{x}}^{\prime}(u)$, where $f_{\bar{x}}^{\prime}$ is the upper Mosco epi-derivative of $f$ at $\bar{x}$.

(ii) If, in addition, $f$ is star-shaped at $\bar{x} \in \operatorname{Dom}(f)$, then $f_{\bar{x}}^{\prime}(u)=\underline{D}_{e}^{R} f(\bar{x} ; u)$ for all $u \in X$.

Proof. Let $\bar{x} \in \operatorname{Dom}(f)$ and put $\bar{y}=f(\bar{x})$. Let $u \in X . f$ is upper epi-differentiable at $\bar{x}, \forall t_{n} \searrow 0$, $\exists u_{n} \rightarrow u$ such that

$$
f_{\bar{x}}^{\prime}(u) \geq \limsup _{n \rightarrow+\infty}\left(\Delta_{t_{n}} f\right)_{\bar{x}}\left(u_{n}\right)
$$

Clearly we have

$$
\underline{D}_{e}^{R} f\left(\bar{x} ; u_{n}\right) \leq f_{-}^{\prime}\left(\bar{x} ; u_{n}\right)=\inf _{t>0}\left(\Delta_{t} f\right)_{\bar{x}}\left(u_{n}\right) \leq\left(\Delta_{t_{n}} f\right)_{\bar{x}}\left(u_{n}\right) .
$$

Thus, in view of the lower semicontinuity of $\underline{D}_{e}^{R} f(\bar{x} ;$.$) (thanks to (3.1) and [3, Theorem 5])$ we obtain, at the limit in (3.2) when $n$ approaches $+\infty$, that

$$
\underline{D}_{e}^{R} f(\bar{x} ; u) \leq \liminf _{n} \underline{D}_{e}^{R} f\left(\bar{x} ; u_{n}\right) \leq \liminf _{n}\left(\Delta_{t_{n}} f\right)_{\bar{x}}\left(u_{n}\right) \leq \limsup _{n}\left(\Delta_{t_{n}} f\right)_{\bar{x}}\left(u_{n}\right) \leq f_{\bar{x}}^{\prime}(u) .
$$

This concludes the first part of the proposition.

Assume now that $f$ is star-shaped at $\bar{x} \in \operatorname{Dom}(f)$ and show that $f_{\bar{x}}^{\prime}(u) \leq \underline{D}_{e}^{R} f(\bar{x} ; u)$. According to Theorem 2.3, we have

$$
\underline{D}_{e}^{R} f(\bar{x} ; u)=\inf _{P}\{v \in \mathbb{R}:(u, v) \in R(\operatorname{epi}(f) ;(\bar{x}, \bar{y})\} .
$$

Let $v \in \mathbb{R}$ s.t $(u, v) \in R($ epi $(f) ;(\bar{x}, \bar{y}))$. Since $f$ is star-shaped at $\bar{x}$, from Lemma 3.2 we obtain that $(u, v) \in T(\operatorname{epi}(f) ;(\bar{x}, \bar{y}))$. Therefore $\exists t_{n} \searrow 0,\left(u_{n}, v_{n}\right) \longrightarrow(u, v)$ such that $(\bar{x}, \bar{y})+t_{n}\left(u_{n}, v_{n}\right) \in$ epi $(f)$. Accordingly,

$$
\left(\Delta_{t_{n}} f\right)_{\bar{x}}\left(u_{n}\right) \leq v_{n}
$$


Hence, combining epi-differentiability of $f$ at $\bar{x}$ with (3.4), it results that

$$
f_{\bar{x}}^{\prime}(u) \leq \liminf _{n \rightarrow+\infty}\left(\Delta_{t_{n}} f\right)_{\bar{x}}\left(u_{n}\right) \leq \lim _{n \rightarrow+\infty} v_{n}=v .
$$

Thus, in view of (3.3), we obtain that

$$
f_{\bar{x}}^{\prime}(u) \leq \underline{D}_{e}^{R} f(\bar{x} ; u) .
$$

This completes the proof.

To establish the upper counter part of Proposition 3.1, we need the following.

Proposition 3.2. Let $f: X \rightarrow \mathbb{R} \cup\{+\infty\}$ and $\bar{x} \in \operatorname{Dom}(f)$. Assume that one of the following assertions hold

i) $f$ is star shaped and upper epi-differentiable at $\bar{x}$;

ii) $f$ is lower epi-differentiable at $\bar{x}$.

Then $f_{\bar{x}}^{\prime}(u) \leq \bar{D}_{e}^{R} f(\bar{x} ; u)$, where $f_{\bar{x}}^{\prime}$ is the lower Mosco epi-derivative of $f$ at $\bar{x}$.

Proof. If $f$ is star shaped and upper epi-differentiable at $\bar{x}$, then the required inequality follows from Proposition 3.1 since every time we have $\underline{D}_{e}^{R} f(\bar{x} ; u) \leq \bar{D}_{e}^{R} f(\bar{x} ; u)$ (thanks to Lemma 2.1). Let us deal with the second case (where $f$ is lower epi-differentiable at $\bar{x}$ ). To do that, let $\bar{x} \in \operatorname{Dom}(f)$ and put $\bar{y}=f(\bar{x})$. Let $u \in X$ and $u_{n} \rightarrow u$. Since $f$ is lower epi-differentiable at $\bar{x}$, $\forall t_{n} \searrow 0$, we have

$$
f_{\bar{x}}^{\prime}(u) \leq \liminf _{n \rightarrow+\infty}\left(\Delta_{t_{n}} f\right)_{\bar{x}}\left(u_{n}\right)
$$

Hence

$$
\left(\Delta_{t_{n}} f\right)_{\bar{x}}\left(u_{n}\right) \leq f_{+}^{\prime}\left(\bar{x} ; u_{n}\right)=\sup _{t>0}\left(\Delta_{t} f\right)_{\bar{x}}\left(u_{n}\right) \leq \bar{D}_{e}^{R} f\left(\bar{x} ; u_{n}\right) .
$$

Thus, in view of the upper semicontinuity of $\bar{D}_{e}^{R} f(\bar{x} ;$.$) (by the use of (3.1) and [3, Theorem 5])$ we obtain, at the limit in (3.7) when $n$ approaches $+\infty$, that

$$
f_{\bar{x}}^{\prime}(u) \leq \liminf _{n \rightarrow+\infty}\left(\Delta_{t_{n}} f\right)_{\bar{x}}\left(u_{n}\right) \leq \limsup _{n \rightarrow+\infty} f_{+}^{\prime}\left(\bar{x} ; u_{n}\right) \leq \limsup _{n \rightarrow+\infty} \bar{D}_{e}^{R} f\left(\bar{x} ; u_{n}\right) \leq \bar{D}_{e}^{R} f(\bar{x} ; u) .
$$

This completes the proof.

\section{AN EXTREMAL VECTOR PROBLEM UNDER INCLUSION CONSTRAINTS}

For any reflexive Banach space $S$, in the sequel we use the following notations:

- $S^{\star}$ for its topological dual and $\langle.,$.$\rangle for its duality pairing;$

- $B_{S}$, for the closed unit ball and $B(\bar{x}, r)$ for the ball centered in $\bar{x}$ with radius $r$;

- $w^{\star}$ for the weak-star topology in $S^{\star}$ and we write $\stackrel{w^{\star}}{\longrightarrow}$ to denote the weak-star convergence in $S^{\star}$ and $\rightarrow$ for the strong convergence in $X$.

In order to express the optimality conditions for problem $(\mathrm{P})$, we introduce the following:

- the support function of $F$ : for $y^{\star} \in Z^{\star}, C_{F}\left(y^{\star}, x\right):=\inf _{y \in F(x)}\left\langle y^{\star}, y\right\rangle$;

- $Z_{F}^{\star}(x)=\left\{y^{\star} \in Z^{\star} \mid C_{F}\left(y^{\star}, x\right)>-\infty\right\}$

- for $x \in X, d_{F}(x):=d(0, F(x))$, the distance from 0 to $F(x)$;

- $I(x):=\left\{y^{\star} \in Z_{F}^{\star}(x) \mid C_{F}\left(y^{\star}, x\right)=d_{F}(x)\right\}$; 
- if the support function $C_{F}\left(y^{\star},.\right)$ is epi-differentiable at $\bar{x}$, we consider the set

$$
D_{F}(x):=\left\{d \in X: \forall y^{\star} \in I(\bar{x}), \forall x^{\star} \in \partial_{e} C_{F}\left(y^{\star}, .\right)(\bar{x}),\left\langle x^{\star}, d\right\rangle \leq 0\right\} .
$$

Recall from [1] the following result, which determines the contingent cone of $F^{-1}(0)$ at a point $\bar{x}$.

Lemma 4.1. Let $\bar{x} \in \operatorname{Dom}(f) \cap \operatorname{Dom}(F)$. Assume the following conditions hold

$\left.C_{1}\right) F$ is Lipshitz at $\bar{x}$ i.e., there exists $\eta>0$ and $r>0$ such that

$$
F(x) \subset F\left(x^{\prime}\right)+\eta\left\|x-x^{\prime}\right\| B_{Z},
$$

for all $x, x^{\prime} \in B(\bar{x}, r)$;

$\left.C_{2}\right) F$ is metrically regular at $\bar{x}$ i.e., for some reals $\lambda, r>0$,

$$
d_{F}(x)=d(0, F(x)) \geq \lambda d\left(x, F^{-1}(0)\right),
$$

for all $x \in B(\bar{x}, r)$;

$\left.C_{3}\right)$ the support function $C_{F}\left(y^{\star},.\right)(x)$ is epi-differentiable and its epi-gradient $\partial_{e} C_{F}\left(y^{\star},.\right)(x)$ is (weak-star/strong)-upper semicontinuous at $\left(y^{\star}, x\right)$ i.e., if $x_{n}^{\star} \in \partial_{e} C_{F}\left(y_{n}^{\star},.\right)\left(x_{n}\right)$ with $x_{n}^{\star} \stackrel{w^{\star}}{\longrightarrow} x^{\star}$ in $X^{\star}, y_{n}^{\star} \stackrel{w^{\star}}{\longrightarrow} y^{\star}$ in $Z^{\star}$ and $x_{n} \rightarrow x$ in $X$, then $x^{\star} \in \partial_{e} C_{F}\left(y^{\star},.\right)(x)$.

Then, the contingent cone of $F^{-1}(0)$ at $\bar{x}$ is given by

$$
T\left(F^{-1}(0), \bar{x}\right)=D_{F}(\bar{x})=\left\{d \in X: \forall y^{\star} \in I(\bar{x}) \mid C_{F}^{\prime}\left(y^{\star}, .\right)_{\bar{x}}(d) \leq 0\right\} .
$$

For a characterization of metric regularity in condition $C_{2}$ ), we refer to [18].

Proof. This result was stated in [1, Lemma 4.4] but its proof is based on conditions of [1, Lemma 4.2] (i.e., $\left.C_{i}\right)$ for $i \in\{1,2,3\}$ ).

In what follows, we still assume that $Y$ satisfies that $(Y, P)$ is a lattice, $P$ is closed, convex, pointed, normal and $\operatorname{Int}(P) \neq \emptyset$, and state the necessary optimality conditions for problem (P).

Theorem 4.1. Let $f: X \longrightarrow Y^{\bullet}, \bar{x} \in \operatorname{Dom}(f) \cap \operatorname{Dom}(F)$ and assume that conditions $\left.C_{i}\right)_{i \in\{1,2,3\}}$ are satisfied and that $\left.C_{4}\right) \underline{D}_{e}^{R} f(\bar{x} ; 0)=0$. Then, if $\bar{x}$ is a solution to $(P)$, then

$$
\underline{D}_{e}^{R} f(\bar{x} ; v) \leq_{P} 0 \forall v \in D_{F}(\bar{x}) .
$$

Proof. Assume that a point $\bar{x} \in \operatorname{Dom}(f) \cap \operatorname{Dom}(F)$ is solution to (P). Let $v \in D_{F}(\bar{x})$. Then by Lemma 4.1, we have $v \in T\left(F^{-1}(0), \bar{x}\right)$. Thus,

$$
\exists t_{n} \searrow 0, \exists v_{n} \rightarrow v \text { s.t } \bar{x}+t_{n} v_{n} \in F^{-1}(0) .
$$

Since $\bar{x}$ is a solution to (P), we have that $f(\bar{x})-f\left(\bar{x}+t_{n} v_{n}\right) \in P^{\bullet}$, which leads to

$$
\left(\Delta_{t_{n}} f\right)_{\bar{x}}\left(v_{n}\right) \leq_{P} 0 \text {. }
$$

Accordingly,

Therefore,

$$
\underline{D}_{e}^{R} f\left(\bar{x} ; v_{n}\right) \leq_{P} f_{-}^{\prime}\left(\bar{x} ; v_{n}\right) \leq_{P}\left(\Delta_{t_{n}} f\right)_{\bar{x}}\left(v_{n}\right) \leq_{P} 0
$$

But in view of Part (b) of Theorem 2.2 and $C_{4}$ ),

$$
\underline{D}_{e}^{R} f\left(\bar{x} ; v_{n}\right) \leq_{P} 0 .
$$

$$
\underline{D}_{e}^{R} f\left(\bar{x} ; v_{n}\right) \neq-\infty \text {. }
$$


It follows that

$$
\underline{D}_{e}^{R} f\left(\bar{x} ; v_{n}\right) \leq_{P} 0 .
$$

Involving the $P$-lower semicontinuity of $\underline{D}_{e}^{R} f(\bar{x} ;$.$) ([3, Theorem 5]) in the last inequality, we$ arrive at

$$
\underline{D}_{e}^{R} f(\bar{x} ; v) \leq_{P} 0
$$

which completes the proof.

Remark 4.1. In the setting of extended real-valued functions $(Y=\mathbb{R})$, the authors of [1], under the additional hypothesis of the epi-differentiability of $f$, proved the following necessary optimality condition:

$$
f_{\bar{x}}^{\prime}(v) \leq 0 \forall v \in D_{F}(\bar{x}),
$$

where $f_{\bar{x}}^{\prime}$ is the Mosco-epi-derivative of $f$. Since (by Part $(i)$ of Proposition 3.1) $\underline{D}_{e}^{R} f(\bar{x} ; v) \leq$ $f_{\bar{x}}^{\prime}(v)$, our optimality condition (4.2) yields more information. Both of the optimality conditions (4.2) and (4.4) coincide for extended real-valued star-shaped functions thanks to Proposition 3.1. Notice that (4.2) has always a sense while (4.4) may not. For example, take simply $Y=\mathbb{R}^{m}$ and $X=\mathbb{R}^{n}, f(x)=A x+b, x \in \mathbb{R}^{n}$, where $A$ is $m \times n$-matrix with real entries, and $b \in \mathbb{R}^{m}$. Then with $P=\mathbb{R}_{+}^{m}$, we have $\underline{D}_{e}^{R} f(\bar{x} ; v)=A v$ for all $v \in \mathbb{R}^{n}$ (see also [7, pp 290]). In this case, we easily see that (4.2) turns out to be $A v \leq_{P} 0$ for all $v \in D_{F}(\bar{x})$, while (4.4) has no sense since Mosco-epi-derivative does not exist beyond real-valued functions, which was the main motivation for us to introduce the alternative of radial epi-derivatives. We also refer to [7, (iii) Remark 3.6] and the reference given therein for another example in which $X=\mathbb{R}, Y=\mathbb{R}^{2}$ and $P=\mathbb{R}_{+}^{2}$.

Theorem 4.2. Let $f: X \longrightarrow Y^{\bullet}, \bar{x} \in \operatorname{Dom}(f) \cap \operatorname{Dom}(F)$ and assume that the conditions $\left.C_{i}\right)_{i \in\{1,2,3\}}$ are satisfied and that $F^{-1}(0)$ is closed and convex. Then, $\bar{x}$ is a solution to $(P)$ if the following condition is satisfied:

$$
\bar{D}_{e}^{R} f(\bar{x} ; v) \leq_{P} 0 \forall v \in D_{F}(\bar{x}) .
$$

Proof. Let $x \in F^{-1}(0)$ and put $v=x-\bar{x}$. Let $\left(x_{n}\right)$ be a sequence in $F^{-1}(0)$ converging to $x$ and $\left(t_{n}\right)_{n}$ a sequence of real numbers in $(0,1)$ such that $t_{n} \searrow 0$. Set $v_{n}=\bar{x}+t_{n}\left(x_{n}-\bar{x}\right)$ to see that $\bar{x}+t_{n} v_{n} \in F^{-1}(0)$ (thanks to convexity of $F^{-1}(0)$ ) and $v_{n} \longrightarrow v$. Thus, $v \in T\left(F^{-1}(0), \bar{x}\right)=D_{F}(\bar{x})$ (by the use of Lemma 4.1). Thus, for every $\alpha>0, \alpha^{-1} v=\alpha^{-1}(x-\bar{x}) \in D_{F}(\bar{x})$. Now by the assumption and Lemma 4.1, we can write

$$
\alpha(f(x)-f(\bar{x})) \leq_{P \bullet} f_{+}^{\prime}\left(\bar{x} ; \alpha^{-1} v\right) \leq_{P} \cdot \bar{D}_{e}^{R} f\left(\bar{x} ; \alpha^{-1} v\right)=\leq_{P} 0 .
$$

This implies that $f(x) \leq_{P} f(\bar{x})$, which ends the proof.

Remark 4.2. Notice that

- Comparing Theorem 4.5 with [1, Theorem 4. 6], we do not require the Lipschitz continuity on $f$ and the setting of the space $X$ is infinite dimensional instead of the finite one. 
- A particular example where the convexity assumption of $F^{-1}(0)$ is satisfied is when $F(x)$ is given by a system of equalities and inequalities defined by a finite family of lower semicontinuous quasi-convex real functions, which is useful in mathematical programming.

- In the proof of the above optimality conditions, the semicontinuity of radial epiderivatives play a crucial role. Notice that, in [7], the radial epiderivatives are not necessarily semicontinuous and the proof as well as the assumption on the ordering cone are quite different.

- According to [3, Remark 9], the above results may be envisaged with a variety of hypotheses on the ordering cone more than the normality or order-completeness.

\section{CONCLUSION}

In this paper, we considered the particular problem of the maximization of a vector-valued map under inclusion constraints. Both necessary and sufficient optimality conditions were presented without any differentiability or Lipschitz continuity condition on the objective functions. Our results recover those of the scalar case and extend some of them to the setting where the feasibility space is an infinite dimensional space with very flexible proof. As noticed in Section 3 and Remark 4.2, such results are applicable to quasi-convex functions, star-shaped functions.

\section{Acknowledgments}

The authors wish to thank an anonymous referee for her/his valuable comments and useful remarks. The primary version of this work was done in the framework of the national thesis doctorate defended by the second author at Chouaib Doukkali university-El Jadida, Morocco, under the joint supervision of the first author and Prof. M. Laghdir to whom the second author wishes to address warm thanks for constant encouragement during this project.

\section{REFERENCES}

[1] T. Amahroq, N. Gadhi, H. Riahi, Epidifferentiability and optimality conditions for an extremal problem under inclusion constraints, J. Inequal. Pure Appl. Math. 4 (2003), Article ID 41.

[2] M. Ait Mansour, H. Riahi, On the cone minima and maxima of directed convex free disposal subsets and applications, Minimax Theory Appl. 1 (2016), 163-195.

[3] M. Ait Mansour, H. Riahi, Extended radial epiderivatives of non-convex vector- valued maps and parametric quasiconvex programming, Optimization 64 (2013), 771-797.

[4] M. Ait Mansour, A. Metrane, M. Théra, Lower semicontinuous regularization for vector valued maps, J. Global Optim. 35 (2006), 155-169.

[5] J. P. Aubin, H. Frankowska, Set-Valued Analysis, Birkhäuser, 1990.

[6] H. Attouch, Variational Convergence for Functions and Operators, Pitman Advanced Publishing, 1984.

[7] F.-F. Bazán, Radial epiderivative and asymptotic functions in non-convex vector optimization, SIAM J. Optim. 14 (2003), 284-305.

[8] C. Combari, M. Laghdir, L. Thibault, Sous-différentiel de fonctions convexes composées, Ann. Sci. Math. Québec, 18 (1994), 119-148.

[9] A. Göpfert, H. Riahi, C. Tammer, C. Zălinescu, Variational methods in partially ordered spaces, SpringerVerlag, New York, 2003.

[10] J. Jahn, Mathematical vector optimization in partially ordered linear spaces, Verlag Peter Lang, Frankfurt am Main, 1986.

[11] J. Jahn, Vector Optimization. Theory, Applications and Extensions, Springer, Berlin, 2004. 
[12] J. Jahn, R. Rauh, Contingent epiderivatives and set-valued optimization, Math. Methods. Oper. Res. 46 (1997), 193-211.

[13] J. Jahn, A. A Khan, Some calculus rules for contingent epiderivatives. Optimization 52 (2003), 113-125.

[14] J. Jahn, A. A Khan, Generalized contingent epiderivatives in set-valued optimization, optimality conditions. Numer. Funct. Anal. Optim. 23 (2002), 807-831.

[15] A. A. Khan, C. Tammer, C. Zălinescu, Set-valued Optimization: An Introduction with Applications, SpringerVerlag Berlin Heidelberg, 2015.

[16] L. Rodríguez-Marín, M. Sama, About contingent epiderivatives, J. Math. Anal. Appl. 327 (2007), 745-762.

[17] M. Sama, Some remarks on the existence and computation of contingent epiderivatives, Nonlinear Anal. 71 (2009), 2997-3007.

[18] B.S. Mordukhovich, Variational analysis and generalized differentiation, I: Basic Theory, II: Applications, Springer-Verlag, 2006.

[19] J-P. Penot, M. Théra, Semi-continuous mappings in general topology, Archiv der Math. 38 (1982), 158-166.

[20] R. T. Rockafellar, First and second-order epi-differentiability in nonlinear programming, Trans. Amer. Math. Soc. 307 (1988), 75-108.

[21] A. Taa, Set-valued derivatives of multifunctions and optimality conditions, Numer. Funct. Anal. Optim. 19 (1998), 121-140. 